\title{
eService Innovation and Sensor Based Healthcare
}

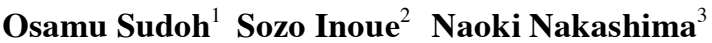 \\ ${ }^{1}$ Graduate School of Interdisciplinary Informatics, Univ. Tokyo, \\ 7-3-1 Hongo, Bunkyo-ku, Tokyo, Japan, 113-0033 \\ sudoh@iii.u-tokyo.ac.jp \\ ${ }^{2}$ Library, Kyushu Univ., \\ 6-10-1 Hakozaki, Higashi-ku, Fukuoka, Japan, 812-8580 \\ sozo@lib.kyushu-u.ac.jp \\ ${ }^{3}$ Kyushu Univ. Hospital, \\ 3-1-1, Maidashi, Higashi-ku, Fukuoka, Japan, 812-8582 \\ nnaoki@info.med.kyushu-u.ac.jp
}

\begin{abstract}
This paper discusses eServices using frontier applications, which targets medical domain applications using sensor network, along with New standardized health check system introduced to Japanese nations recently. We clarify the ubiquitous aspects of this application, and address challenges for ubiquitous innovation. We also describe the experience of introducing and operating the system with a real field trial.
\end{abstract}

\section{Introduction}

There is growing momentum today around the world to encourage innovations based on information networks. As defined by Joseph A. Shumpeter in The Theory of Economic Development in 1926, innovation is the creation of value through unprecedented combinations of the means of production, resources, the workforce and other components. It is open innovation that is now rapidly proliferating around the globe. Unlike innovation based solely on internal resources, open innovation involves collaboration among multiple players, making effective use of external resources on the basis of networks. In other words, new value is created not by a single unusual talent

Please use the following format when citing this chapter:

Sudoh, O., Inoue, S., Nakajima, N., 2008, in IFIP International Federation for Information Processing, Volume 286; Towards Sustainable Society on Ubiquitous Networks, eds. Oya, M., Uda, R., Yasunobu, C., (Boston: Springer), pp. 1-14. 
but by stepping up interaction among multiple players that are able to work with latent creative abilities. The architecture of participation and interaction is therefore the key to open innovation.

From the perspective of open innovation discussed above, all organizations including administrative, medical, educational and research bodies will need to make fundamental considerations. So this paper discusses New Public eServices using frontier applications, especially Medical eService using sensor network.

\section{Open Innovation and eServices}

It is necessary for us to envision a new paradigm for open innovation which will be utilized to achieve sustainable social development. In the new paradigm, eGovernment and Local e-government should be emphasized as a core function. The infrastructure must be developed into a platform so that it can be applied to the entire society.

Joint outsourcing helps multiple government agencies and local bodies utilize common information systems. However, each information system has a number of identical software modules. In the framework of common infrastructure for eGovernment and Local e-government built based upon SOA (Service Oriented Architecture), common functions are built into modules so that efficiency and transparency in administrative systems can be developed. The following section explains social transformation via a wider-area collaboration of Local e-government.

SOA is a group of independent software modules, each of which is structured to support the requirements of a certain job process and/or common functionality across entire job functions. They are reusable and distributed over the network. The relationships against the modules are assigned by controllers to suit overall system architecture. Duplicate investment can be avoided with SOA, and a drastic decrease in cost can be achieved.

The concept of Local e-government can be strategically developed for the regional growth of the society. SOA is used to modularize software applications which will have varied usage not only for administrative processes but also for administrations in public facilities, hospitals, schools, and day care centers. The outsourcing of SOAbased systems with data centers will greatly contribute to the performance improvement of local SMEs.

The concept of e-Government initiatives can be a driving force for complete transformation of the regional community. To achieve this goal, it is important for us to utilize not only administrative information systems but also medical, welfare, educational institutions and facilities to actively utilize information facilities and infrastructure. Even local private corporations and households should participate.

In many advanced countries, aging society has become a serious social issue. In this context, it is important to prevent the increase of medical costs and to consider preventive health. Various research projects for preventive medicine utilizing IT are currently underway in the world.

We have also taken part of the development of a health management test bed using sensor networks, which is to serve as an empirical research platform. The primary 
goal of the research is to organize a preventive health system to curb deterioration due to various adult diseases, such as high blood pressure, diabetes, kidney disease, and cardiac illness, to prevent complications, and to support secondary prevention for the improvement of health condition. Wearable bio-sensors and earthed indoor sensors are in place to obtain patient's ecological data. The data is transmitted from Mote client to the data center via IP-VPN. Data is made anonymous before being sent over remote grids. Advanced data analysis is performed for a large volume of collected data pertaining to body movements, and is reported by the system to designated doctors. This is to support medical diagnosis and consultation by doctors for health management.

If the research produces certain outcomes, it will be upgraded into a larger empirical initiative using the research infrastructure of the sensor network and ASP, which is used to introduce preventive health services and security services.

Several leading municipalities are developing SOA-based information infrastructure in a joint effort. The Japanese medical insurance system has been reformed recently to prevent the increase of medical costs, through health prevention and management efforts in local communities. Therefore, the management of medical and health-related information at the community level has become an important issue. In this regard, it is possible to take a strategic approach to the evolution of local communities by incorporating the community model based on the sensor network infrastructure into the entire Local e-government scheme.

The SOA concept is being promoted by these leading local governments, in which software is modularized and used for a variety of purposes. Software modules are not only for government administration but also public facilities, hospitals, and daycare centers. If the aforementioned local health management architecture using sensor networks is linked to this joint SOA initiative, the local information infrastructure would increase optimal efficiency in management of medical and welfare facilities where new quality services could be created.

This paper will show the innovative capability of medical eService that should be based on SOA and ASP.

\section{Healthcare}

\subsection{Background}

The results of the national nutrition survey study in 2006, conducted by the Ministry of Health, Labour and Welfare, showed that 8.2 million people have diabetes, and if those in the diabetes-prone group are added, that figure would be 18.7 million people among the total Japanese population of 127 million people. Along with the increase in diabetes, there have also been alarming increases in other diseases caused by had life-style habits hypertension and dyslipidemia. Approximately 39.7 million have hypertension and 14.1 million have dyslipidemia. These three diseases are considered 
to be major causative factors in the two diseases that account for the second and third ranking causes of death in Japan, myocardial infarction and brain stroke, respectively. Additionally, uncontrolled diabetes causes chronic renal failure, and leads hemodialysis, which spends 5 million yen per year x person. Recent studies have shown that obesity with visceral fat accumulation, which is commonly known as "metabolic syndrome", is the main cause of diabetes, hypertension and dyslipidemia. The survey shows 19.4 million have metabolic syndrome if those in prone group are added. That means that this is an age in which we must call metabolic syndrome the national disease.

In the typical course, obesity begins in their 20's to 40 's, developing to lifestyle diseases in their 40's to 60's. As the results, elderly people spend higher medical cost by causing atherosclerotic diseases, or by hemodialysis. Younger onset of these serious diseases leads to the decrease of working resources.

Accordingly, in order to avoid contracting these life threatening diseases, to inhibit increasing medical cost, and to keep productivity, the first issue of priority is the prevention of obesity and the control of metabolic syndrome.

\section{New Standardized Health Check-up System (Particular Health Check-up Sys- tem; PHCS)}

In June 2006, the Japanese government amended the Medical Care Law to establish an annual health check-up/management system for all citizens aged $40-74$ years. This system will greatly affect insurers because it will cover 57 million citizens and will involve all insurers from April 2008. Insurers will be penalized economically from 2013 when stated goals are not achieved. All Japanese have basic health care insurance.

The annual health check-up is described as follows:

1. Questionnaire (inquiry pertaining to weight change, smoking, exercise).

2. Physical examination (height, weight [body mass index], waist, blood pressure).

3. Blood chemistry tests (Triglyceride, HDL-cholesterol, LDL-cholesterol, GOT, GPT, $\gamma$-GTP, Cre, blood glucose (fasting or postprandial), HbA1c, uric acid).

Stratification involves two steps. On the basis of results, participants are assigned to one of three risk groups. The waist and body mass index determine the first assignment. Risk factors identified by blood chemistry tests and smoking history are tallied, and participants are assigned to the "information provided group", "motivation support group", or the "aggressive support group" (Fig.1). 


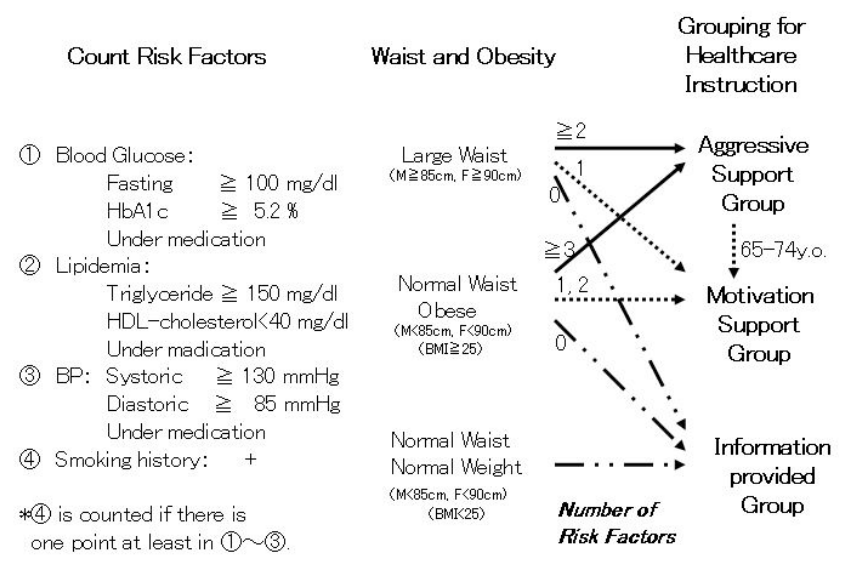

\section{Fig.1 Stratification into risk groups according to the results of physical exami- nation and blood chemistry tests (ref.[5]).}

Insurers need to provide healthcare instruction once to individuals assigned to the motivation support group, and repeatedly to individuals assign to the aggressive support group. The information provided group is not given health instruction. A physician, a health nurse, or a registered dietitian should provide the first healthcare instruction each year. Non-face-to-face instruction via telephone or information and communication technology (ICT) system is allowed for additional instruction on the aggressive support group. Insurers do not need to provide healthcare instruction to the person who is under medication of lifestyle disease when he/she is classified into the motivation support group or the aggressive support group.

All data obtained from the PHCS will be digitized in a standard protocol as HL7 CDA and in standard cords as JLAC 10. Insurers will have to maintain records for each insured person as long as he or she remains insured. The records will contain health check-up and instruction data. If the individual changes insurer as he changes occupation, the former insurers will transfer the data to the new insurer so that there is no gap in the record. With these systems in place, it is anticipated that the Japanese government have access to huge amounts of anonymous data that can be used for statistical purposes. Insurers can keep the data without anonymising it because, for example, they have to analyze it together with the receipt data from the medical institutions. Insurers will be able to store data more effectively when the on-line reimbursement project is fully achieved in 2011 .

The Japanese government suggests that the insurer pay an additional $10 \%$ contribution to medical cost of aged person (age 75 year or over) if it does not achieve the stated goals or be rewarded by a $10 \%$ discount if stated goals are achieved from 2013. The Japanese government is asking for a $25 \%$ decrease in the number of diabetes and pre-diabetes patients per insurer for example.

Insurers will be allowed to outsource the required tasks to healthcare provider companies. Thus, many tasks will be outsourced to Internet data centers.

The initial action of insurers will be the engagement of a health-care provider company for outsourcing. After registration of the covered citizens, the health-care 
provider will begin service. If the providers do not produce adequate outcomes, insurers must pay the penalty. Insurers must take overall responsibility.

\section{Our Research field, "Carna"}

A venture company Carna Health Support participated as an outsourcing company for data management with IT system and healthcare instruction. Carna Health Support originally launched to establish a Japanese model of disease management in diabetic field in 2006 by diabetic specialists in Kyushu University and collaborator companies. Thus, Carna has two programs, one is the primary prevention program for PHCS, and another is the secondary/tertiary prevention program for diabetes mellitus.

To motivate clinics and patients to participate in disease management, Carna supports the family doctor system and education for patients.

For clinics, Carna matches doctors and new patients when individuals are affected with a lifestyle disease. Carna also attempts to prevent patient dropout by telephone contact, support patient education and detection of early-stage complications by means of periodic questionnaires, and facilitate medical specialists' cooperation according to the timing described in the critical pathway system.

For patients, Carna promotes guideline medical care described in the critical pathway, report the results of blood tests quickly, and provide 'Carna points' as rewards for the patient's efforts (for instance, regular clinic visits) and for improvement in their diabetic condition (HbA1c). Carna exchanges the points for coupons with which they can obtain certified health-related products such as healthy foods and exercise goods.

(1) Critical pathways for quality control, for appropriate matching of services to individuals, and for adaptation to the political direction in Japan.

Carna developed a region-related, outcome-oriented critical pathway as the core competency in the call center. Carna also standardized workflow in the call center calling 'algorithm'. The critical pathways and the parts of the algorithm are digitized. Carna prepared an education system with structured questionnaires and comprehensive teaching materials, which are closely related to the personalized critical pathway.

Carna had two kinds of outcome-oriented critical pathways by the end of 2006. One is for the primary prevention program (lifestyle improvement program). The other is for the secondary/tertiary prevention program for diabetes mellitus.

Features of the critical pathway for lifestyle improvement program edge are described below;

- Using five kinds of critical pathway matching each stage of Prochaska stage model (pre-contemplation stage, contemplation stage, preparation stage, action stage, maintenance stage) .

- Matching the framework of the PHCS

Features of the critical pathway for diabetes mellitus (secondary/tertiary prevention) that Carna has developed are described below;

- Scheduling of medical services based on clinical guidelines produced by the Japanese Diabetic Society. 
- Supporting general care of diabetic outpatients including timely reminders of the need to visit medical specialists such as an ophthalmologist, and a diabetologist.

- Using 'the overlay method' to create an optimal personalized critical pathway for each patient. A personalized critical pathway is created by overlay with a basic sheet for regular examination, and optional sheets matching patient's treatments, the severity of the diabetic complications, and the patient's level of knowledge. Carna can create 2,880 different of critical pathways.

- Modifying continuously as the patient's condition

(2) Personalized communication based-on patient characteristics

Patients' responses to interventions vary because the basic characters of patients vary. For successful intervention, Carna determines the patient's character type during the registration process, and Carna depends on this information to personalize our communication with the patient. This approach may also decrease stress of the call center staff.

(3) Other algorithms and ICT system for efficient and secure data management

Carna developed other algorithms ICT system as shown in Fig.2. Carna used application service provider system for ICT to input participant records.

Carna is using an Internet data center in Fukuoka city, Japan, for database servers. Carna sends data over the Internet via virtual private network from the call center. 


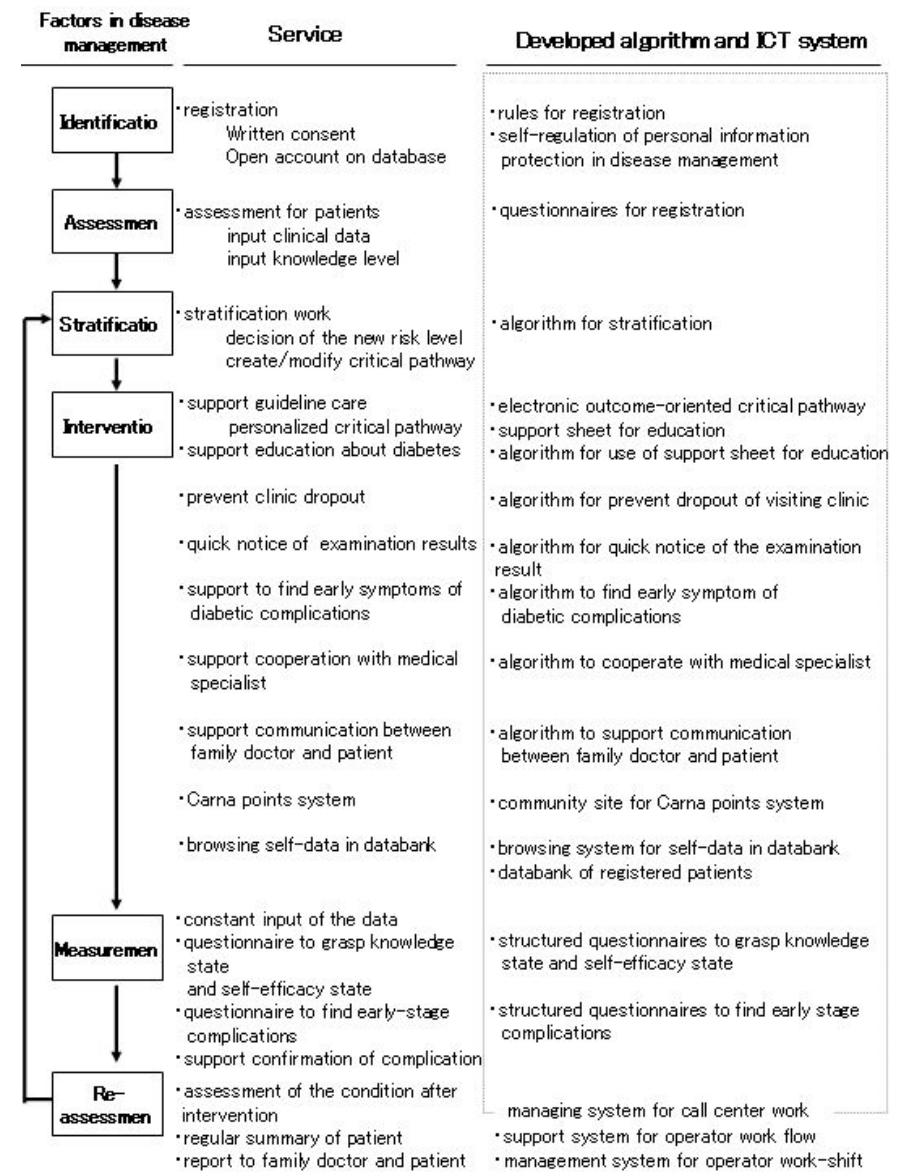

Fig. 2 Disease management services and rules, algorithms, and ICT system in the secondary/tertiary prevention program for diabetes mellitus (ref.[5])

(4) Ethical considerations

Carna quantified the risk of disclosure in terms of information value, threat arising from inadequate ICT security, and areas of vulnerability and showed that the highest risk was posed by databases containing individual patient profiles. Consequently, We believes that we need regulations pertaining to the provision of health information and that we need general classifications for various types of patient information that we deal with in disease management work.

\subsection{SOA Based Healthcare using Sensor Network}

We aim to develop a SOA based healthcare system of Canra which manages effective PHCS, integrated with sensor networks. Using sensor network with vital sensors, the activities and lifestyles of patients/citizens can collected in an easy and accurate manner, and therefore effective healthcare can be possible. 
However, since the entire system will be highly complex system, the following issues have to be considered.

\section{Scalability}

Since the medical check system is to be applied to massive populations in long term, the system has to handle huge amount of data. Distributed architecture which can process massive data will be needed. Especially, maintenance cost in an wide area distribution should be seriously considered.

\section{Heterogeneity}

The system should handle various kinds of data, such as manually input data, sequential motion sensor data, and periodical vital sensor data. Along with this, sensors and networking devices will also be heterogeneous to meet with the varieties of required data and reliability.

\section{Reliability}

Because of the variety of data and of the requirement for long battery life of mobile sensor devices, there should appear trade-offs between accuracy of data and energy consumption. For example, we can analyze the movement of an patient more accurately and more in real time if we could obtain frequent motion data from an accelerometer, but it results in severe battery life. Optimal balance of these parameters should be discovered for reliability of data.

\section{Human Friendliness}

Since the system is to be used in the daily lives of patients, it should be highly user-friendly as much as stress free to patients. It has the following two aspects:

- Human interfaces such as web interfaces should be easy to use, and easy to be embedded to the patients' lifestyles, so as in the case of blog systems.

- Vital sensor devices should be non-intrusive, small as much as possible, and even free in the way of attaching to the body. For example, a heart rate sensor attached to the finger keeps the patient away from desk work.

\section{Security and Privacy Management}

The data handled in the system are sometimes highly privacy-sensitive ones. Therefore, complete security and adequate personal data access control are the necessary.

\section{Flexibility}

This is the most important feature. Although we addressed the above requirement for the system, we still do not know how much we should decide the qualitative or quantitative parameters or implementations from so many design options.

It means that we fundamentally need many experiences and trials, that we should adopt the style of rapid development based on the feedbacks from the trials. Here, the reason why SOA, which realizes highly modularized composition of service functions, is crucial in this healthcare domain. 


\section{Experience}

In this section, the experience aimed for effective medical check systems are described. In the experience, sensor network was introduced for automatically monitoring the activity of patients, and for advising the patients adequately. Moreover, the obtained data from the sensors as well as manually recorded data can be used for medical knowledge discovery which is unknown so far.

\subsection{Design}

For simplicity, we classify the users of the system into two roles: patients, and data analyst. Patients are the users who resulted in the need for continuous care by specific medical check. Data analysts are the users who analyze the data from the sensors along with the manually recorded data, and correspond to physician, health adviser in the specific medical check system, and medical researcher in actual situation.

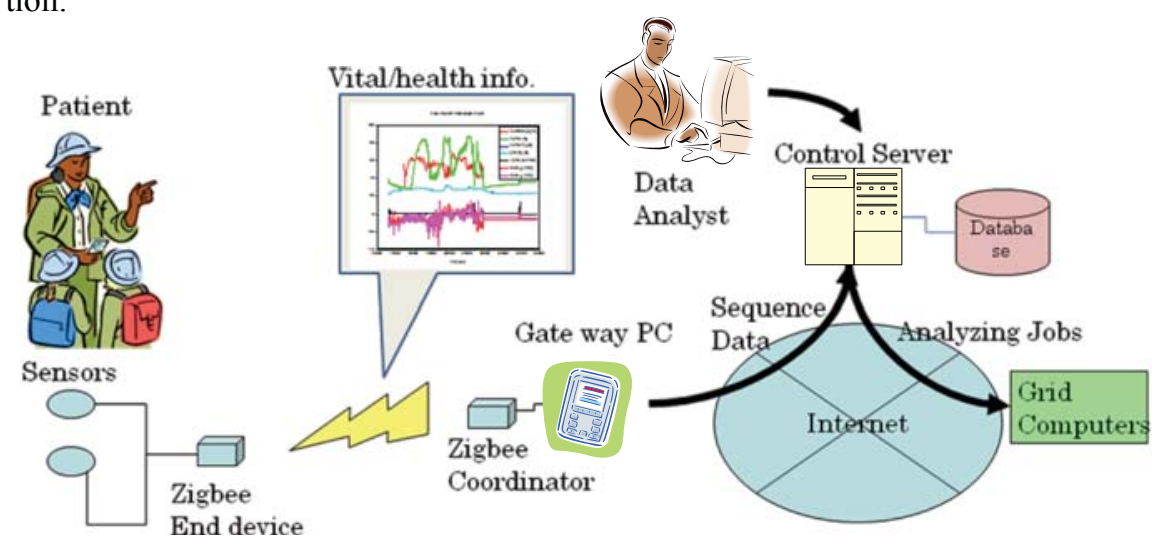

Fig.3 System and Network Architecture

Fig. 3 is the system and network architecture of the system. The system consists of sensors, zigbee[8] end devices, zigbee coordinator, gateway PC, control server, database, and grid computers. The use cases of the system are described in the following.

\section{Patient}

Patients have sensor end devices in their lives. End devices attached with sensors acquire vital data of the patients periodically, and send them to zigbee coordinator through routers.

Routers, coordinators, and gateways are placed in offices and homes, and receive data from the end devices, and send to the control server. Gateway PCs also accept manual inputs from the patients. 


\section{Data Analyst}

Data analysts do data analysis, or data mining using the data gathered to the control server.

The control server stores the data from gateway PCs to the database systems, and throws analysis/mining jobs by data analysts to grid computers, with the data, and receives the answer.

\subsection{Consequence}

Based on the architecture introduced above, we tried a first-step experience, where basic design options, such as communication protocols, communication throughput, human friendliness of sensor devices, sensor measurement methods, are discovered with reasonably small amount of population in a single place.

The trial was done in a building which has a hospital and a fitness club at once. 3 patients participated at one time, and repeated 3 times for independent groups, which means 9 patients in total.

The trial was scheduled as the following:

$1^{\text {st }}$ day: medical check (Fig. 4(a))

In the first day, the patients took full-fledged medical check, including blood, urinalysis, ECG, X-ray, body consumption, BMI, blood pressure, and CPX(cardiopulmonary exercise).

Especially, CPX (Fig.4(a)) has an important factor. In CPX, an adequate heart rate can be calculated from variety of measurements such as the rate of $\mathrm{O} 2$ and $\mathrm{CO} 2$ in breath during an exercise. This value of heart rate can be used in the exercises in the following days.

$2^{\text {nd }} \sim 7^{\text {th }}$ day: exercise and dietary program (Fig.4 (b))

From the next day, the patients take fitness exercises, including body stretch, aerobic exercise, and muscle training, for about 2 hours.

Besides, the patients are managed dietary program. 2 of the meals in a day are designated by a dietitian, and diet soup and diet frozen food are sent to the home of the patients. And what they ate in the rest of the day are interviewed and recorded by the dietitian. To be accurate, this is done from the $1^{\text {st }}$ day.

$8^{\text {th }}$ day: measurement of the effects (Fig.4(c) )

After the days of exercises and dietary program, the effect of the exercises and diets are evaluated by measuring the change of blood factors, urinalysis, body composition, BMI, and blood pressure. 


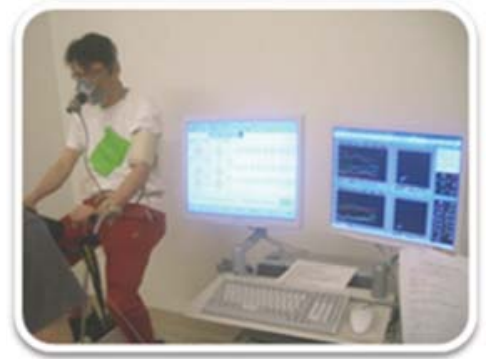

(a) $1^{\text {st }}$ day

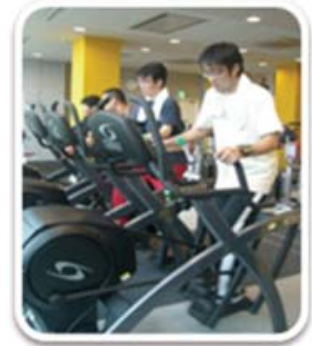

(b) $2^{\text {nd }} \sim 7^{\text {th }}$ day

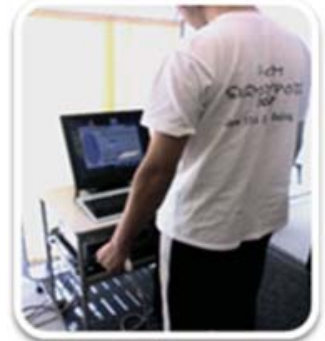

(c) $8^{\text {th }}$ day

\section{Fig. 4 Scene of trial}

\section{Sensor and zigbee devices}

For this trial, we used the following sensor devices

- $2 \mathrm{D} / 3 \mathrm{D}$ accelerometers

- Temperature, humidity, and light sensors

- Heart rate sensors (non-zigbee)

- Heart rate sensors (non-wireless)

Fig. shows one type of the sensor zigbee devices, which has 2D accelerometers, temperature, humidity, and light sensors, and gateway PC.

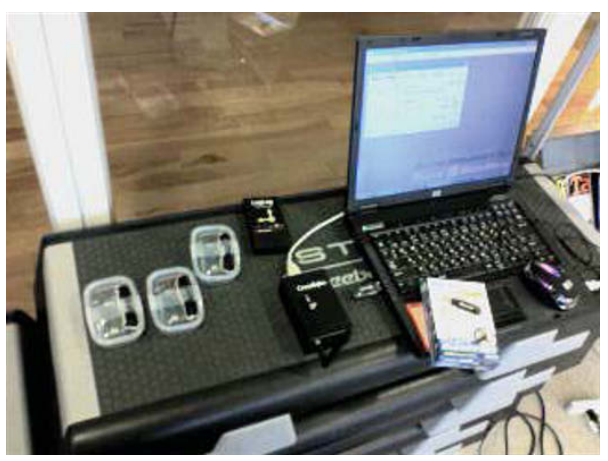

Fig. 5 Sensor Zigbee devices and gateway PC

\section{Obtained Data}

Through the trial, obtained data are the following:

- Medical check of the $1^{\text {st }}$ day, such as blood tests, body/athletic abilities, body compositions, urinalysis, and thoracic pictures,

- CPX data of the $1^{\text {st }}$ day(

- Lifestyle related inqury to the patients

- Record of excercises from $2^{\text {nd }}$ to $7^{\text {th }}$ days, such as weight of before/after, blood pressure of before/after, and, time, number, expected calorie of each exercise item.

- Sensor data of exercise hours(

- Calorie intake during the dietary program, and,

- The change of blood test, BMI, body composition in the $8^{\text {th }}$ day.

After all, detailed data for analysis or data mining could be successfully obtained through the trial. 


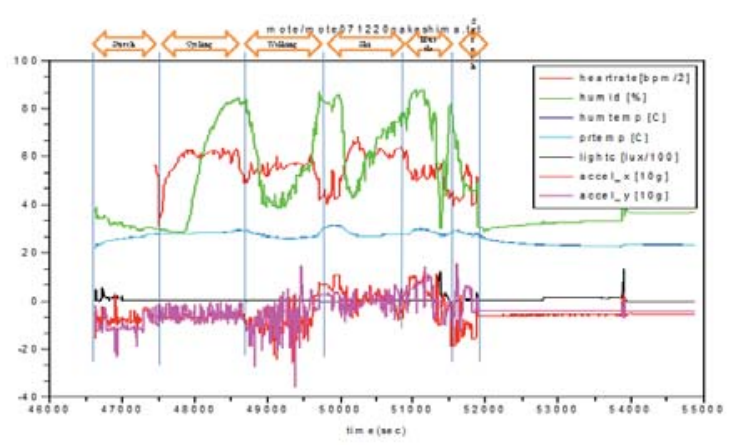

Fig. 6 Example of sensor data

\section{Conclusion}

Here, we discuss the aspects of SOA in the sensor based medical domain we experienced.

First of all, SOA is often discussed in the context of web systems, which means online and homogeneous system. However, we tried ubiquitous environment where network is not always online, and many kinds of small devices which sometimes has no programmability to developers. It addresses many future challenges to SOA environments.

For scalability, we acknowledged the ease of maintenance of zigbee protocol. Multi-hop communication can extend the communication area, and easy handshake protocol reduces the time and maintenance cost of reconnect.

For heterogeneity, the only device types we could adapt to was one which has open source OS, and on site functionality of updating software. This kind of flexibility must be required although it might affect the device cost and power consumption.

For reliability, we could reduce the sampling frequency of accelerometer to several $\mathrm{Hz}$ while keeping the accuracy of activity recognition, which leaded to the longer battery life.

For human friendliness, not only intrusive sensors which we did not use, but also types of sensors which had to be fixed on specific places of a body, such as a heart rate sensor on the chest or on the finger, and an accelerometer which was designated to be fixed on the back, was hated by patients. On the other hands, sensors which are designated to be placed in any place on the body such as a pocket, were easier to use, while they reported practically accurate data, such as an accelerometer, temperature, and humidity sensors.

Security and privacy management, and flexibility on the server side are the future challenges to be discussed to fit SOA. 


\section{Acknowledgement}

This work is a part of Info-plosion: Cyber Infrastructure for the Informationexplosion Era, supported by Grant-in-Aid for Scientific Research on Priority Areas, MEXT, Japan.

\section{References}

[1] Council on Competitiveness ed. (2005) Innovate America, Council on Competitiveness US.

[2] Gregory G. Curtin, Michael H. Sommer and Veronika Vis-Sommer eds. (2003) The World of E-Government, The Haworth Press

[3] Japanese Ministry of Health, Labour and Welfare: Particular health checkup/instruction, http://www.mhlw.go.jp/bunya/kenkou/seikatsu/pdf/02.pdf

[4] Kobayashi, K., Nakashima, N., Inoguchi, T., Nishida, D., Tanaka, N., Hoshino, A., Hamada, M., Kozuma, Y., Nakaguma, H., Matsushita, R., Soejima, H., Takayanagi, R., Nawata, H.(2006) Development of a new critical pathway for regional medical network for outpatients with diabetes mellitus. J. Japan Diab. Soc. 49: 817 - 824.

[5] Nakashima, N., Kobayashi, K., Inoguchi, T., Nishida, D., Tanaka, N., Nakazono, H., Hoshino, A., Soejima, H., Takayanagi, R., Nawata, H.(2007) “A Japanese Model of Disease Management," Stud Health Technol Inform, 129: 1174-8.

[6] Sudoh, O. (2007) Community Governance and Sensor Network in the Era of Info-Explosion, Tongji University ed., Proceedings of International Symposium on Urban Governance and Community Development, Shanghai, pp.161-183

[7] Sudoh, O. ed. (2005) Digital Economy and Social Design, Springer-Verlag

[8] Zigbee Alliance, http://www.zigbee.org/ 